THE RATIONAL CONTINUED INFLUENCE OF MISINFORMATION

The rational continued influence of misinformation

Saoirse A. Connor Desai1, Jens K. Madsen2, Toby D. Pilditch3, 4

University of New South Wales1, London School of Economics2, University of Oxford3, \& University College London4

$6 *$ Correspondence concerning this paper should be sent to Saoirse Connor Desai, School of

7 Psychology, University of New South Wales, 1006, Mathews Building, 8, Kensington NSW

8 2052; Email: saoirse.c.d@gmail.com

9

10 Supplementary materials and data are available at https://osf.io/6yq47 


\section{THE RATIONAL CONTINUED INFLUENCE OF MISINFORMATION}

\section{Abstract}

16 Misinformation has become an increasingly topical field of research. Studies on the 'Continued

17 Influence Effect' (CIE) show that misinformation continues to influence reasoning despite

18 subsequent retraction. Current explanatory theories of the CIE tacitly assume continued reliance

19 on misinformation is the consequence of a biased process. In the present work, we show why this

20 perspective may be erroneous. Using a Bayesian formalism, we conceptualize the CIE as a scenario

21 involving contradictory testimonies and incorporate the previously overlooked factors of the

22 temporal dependence (misinformation precedes its retraction) between, and the perceived

23 reliability of, misinforming and retracting sources. When considering such factors, we show the

24 CIE to have normative backing. We demonstrate that, on aggregate, lay reasoners $(\mathrm{N}=101)$

25 intuitively endorse the necessary assumptions that demarcate CIE as a rational process, still exhibit

26 the standard effect, and appropriately penalize the reliability of contradicting sources. Individual-

27 level analyses revealed that although many participants endorsed assumptions for a rational CIE,

28 very few were able to execute the complex model update that the Bayesian model entails. In sum,

29 we provide a novel illustration of the pervasive influence of misinformation as the consequence of 30 a rational process.

31 Keywords: Continued Influence Effect; Negation; Reliability; Dependency; Reasoning 


\section{THE RATIONAL CONTINUED INFLUENCE OF MISINFORMATION}

\section{Introduction}

The harmful effects of misinformation have become a significant concern in

34 contemporary society (Lewandowsky et al., 2017). These concerns are in part due to the ways

35 that misinformation can spread rapidly online, as the news industry and general population alike,

36 can share information outside of traditional information outlets. Media outlets can report false or

37 inaccurate details while newsworthy events are still unfolding: as demonstrated when a

38 prominent daily newspaper broke an online story incorrectly claiming Russian hackers had

39 penetrated the US electricity grid. When, in fact, the electricity utility at the centre of the story

40 found malware connected with Russian hackers on a single laptop, unconnected to the grid.

41 Although the newspaper updated its article within a few hours of its original post, the incorrect

42 information had already ricocheted through social media and the global news environment.

43 Errors such as these are particularly worrying because several studies have shown that even clear

44 and credible corrections often fail to eliminate the effects of misinformation (see Lewandowsky

45 et al., 2012 for review). This phenomenon is known as the Continued Influence Effect (CIE) of

46 misinformation (Ecker et al., 2010; Johnson \& Seifert, 1994).

47 Continued influence studies examine corrections 1 to misinformation using variants of a

48 laboratory paradigm first developed by Wilkes and Leatherbarrow (1988; but see also Johnson \&

49 Seifert, 1994). In a typical CIE experiment, participants read a fictitious news report presented as

50 a series of sequential statements. Misinformation, offering a causal explanation for the event's

51 outcome, is presented and retracted. A subsequent comprehension test typically shows that

1 We use 'correction' and 'retraction' interchangeably throughout. 


\section{THE RATIONAL CONTINUED INFLUENCE OF MISINFORMATION}

52 misinformation continues to influence memory and inferences even when participants understand

53 and remember the retraction.

One scenario commonly used in the CIE paradigm concerns a warehouse fire in which

55 initial reports suggest that flammable chemicals carelessly stored in a closet caused the fire

56 (Connor Desai \& Reimers, 2019; Ecker, Lewandowsky, Swire, et al., 2011; Guillory \& Geraci,

57 2010; Johnson \& Seifert, 1994; Wilkes \& Leatherbarrow, 1988; Wilkes \& Reynolds, 1999). One

58 group of participants receive a retraction stating that "the closet was empty before the fire"

59 thereby contradicting earlier misinformation. Retraction group responses are typically compared

60 to a control group for whom there was no retraction or a group who were never exposed to the

61 misinformation. The key CIE finding is that retractions are only partially effective: a retraction

62 either results in no difference compared to no retraction (Johnson \& Seifert, 1994), or reduces

63 but fails to eliminate the misinformation's influence (Ecker, Lewandowsky, \& Apai, 2011;

64 Ecker, Lewandowsky, Swire, et al., 2011; Ecker et al., 2010; Guillory \& Geraci, 2010, 2013;

65 Rich \& Zaragoza, 2016).

To date, there have been two leading cognitive explanations for the CIE (Gordon,

67 Brooks, Quadflieg, Ecker, \& Lewandowsky, 2017; Lewandowsky et al., 2012). The selective-

68 retrieval account suggests that the CIE occurs when there is simultaneous storage of correct and

69 incorrect information in memory; upon retrieval, misinformation is activated but inadequately

70 suppressed (Ecker, Lewandowsky, Swire, et al., 2011). The model-updating account instead

71 argues that corrections are poorly encoded because correcting misinformation leaves a gap in

72 people's mental model of the described event. Misinformation is therefore maintained because

73 people prefer a coherent (incorrect) to an incomplete (correct) mental model. People are often

74 unable to fill the gap in their mental-model, left by correcting misinformation unless a correction 


\section{THE RATIONAL CONTINUED INFLUENCE OF MISINFORMATION}

75 offers an alternative explanation for the outcome of the event (Connor Desai \& Reimers, 2019;

76 Ecker, Lewandowsky, \& Apai, 2011; Ecker et al., 2010; Johnson \& Seifert, 1994; Rich \&

77 Zaragoza, 2016). While selective-retrieval relates the CIE to and the inadequate suppression of

78 misinformation at retrieval, the model-updating account posits a failure to update the mental-

79 model stored in memory.

80 The selective-retrieval and model-updating accounts tacitly assume that the CIE is an

81 error, or that normatively, a correction should reduce reliance on misinformation to the same

82 level as would be observed if there was no misinformation at all. Both accounts presuppose that

83 it is always appropriate to disregard earlier 'incorrect' information in favour of the subsequent

84 presented 'correct' information. For this assumption to hold, the 'correct' information must

85 sufficiently 'cancel out' the original 'incorrect' information. In this paper, as explained below,

86 we explore the possibility for a rational foundation for the CIE, which considers the temporal

87 dependence between misinformation and its subsequent correction, and the perceived reliability

88 of the misinforming and retracting sources.

\subsection{Temporal Dependence and Continued Influence of Misinformation}

90 In this paper, we conceptualize the CIE as a scenario involving a contradiction between

91 the testimonies of misinforming and retracting sources. As mentioned previously, there are two

92 assumptions necessary for the retraction to 'cancel out' the misinformation: 1) the retracting

93 source (either the same source at a later time-point or a different source at a later time-point) is

94 perceived to be at least as reliable as the misinforming source, and 2) the sources make their

95 reports independently from one another. That is, the source of the retracting report bears no

96 relation to the misinforming source, whether by sharing evidence (Schum, 1994), or background

97 (Bovens \& Hartmann, 2003; Madsen et al., 2020). When conceptualized as a matter of 


\section{THE RATIONAL CONTINUED INFLUENCE OF MISINFORMATION}

98 contradicting testimonies, the CIE could reflect a dependency effect. In this scenario, the

99 observing reasoner perceives one source which contradicts itself or two sources that contradict

100 each other, as less reliable due to the inconsistency between their first and second reports. When

101 a source contradicts their earlier statement, the reasoner does not know which testimony is truly

102 correct but knows a source has been openly wrong on at least one occasion and therefore

103 penalizes the reliability of all reporting sources. Crucially, in situations where two sources do not

104 know what the other has said (i.e. the sources are conditionally independent), yet provide

105 contradictory reports, then the two reports should cancel out. In such a case, there should be no

106 CIE. However, if the source making the retraction is aware of what the source of the

107 misinformation has said (as is usually the case when a retraction is issued), the sources are no

108 longer independent. More precisely, there is an asymmetry between the original and correcting

109 reports: the strength of the correcting report is not just a product of the reliability of the

110 correcting source but is also a function of how the correcting source responds to the original

111 (misinforming) report, whereas the reverse is not true for the original (misinforming) reporter.

112 Depending on the assumptions outlined above, this inconsistency can produce a difference in the

113 reliability penalty applied to each source, given they contradict each other. Specifically, the

114 corrector (second source) is penalized more than the misinformer (first source), and as such the

115 correcting testimony is deemed weaker, and a continued belief in the (misinformation)

116 hypothesis remains (i.e. a CIE).

117 Work in evidential reasoning on testimony has illustrated the necessity of the above

118 assumptions (Hahn et al., 2009; Hahn, Harris, et al., 2013; Hahn, Oaksford, et al., 2013; Schum,

119 1994; Schum \& Martin, 1982) for disagreement (misinformation vs correction) to have a

120 nullifying effect. In order for independence to hold in the CIE case, the 'corrector' cannot be 


\section{THE RATIONAL CONTINUED INFLUENCE OF MISINFORMATION}

121 aware of the misinformation they are correcting - impossible in the same source case, and

122 unlikely in the different source case. Instead, we must consider there to be a 'temporal'

123 dependency between the two pieces of information because the misinformation precedes its

124 correction - something known to influence the reliability of sources providing evidence for a

125 hypothesis (Madsen, Hahn, \& Pilditch, 2018; 2019).

126 When considering the temporal dependence between misinformation and its retraction,

127 and the reliability of misinforming and retracting sources, we seek to shed new light onto an

128 effect that has, to date, escaped any normative account of how people should process corrections

129 to misinformation. Indeed, sensitivity to temporal order has been previously shown to affect the

130 CIE wherein retractions followed by valid information/explanation are more effective than

131 retractions preceded by valid information (Ecker et al., 2015). However, the temporal

132 dependence between misinformation and its correction, and the impact of this temporal

133 dependence on perceived source reliability, have yet to be considered within a formal

134 framework.

135 In this paper, we formalize the CIE within a Bayesian Network (BN) model (Pearl, 1988)

136 to test whether there may be a rational explanation for the CIE. Bayesian Networks use graph

137 structures to represent probabilistic relations between hypotheses and evidence, showing which

138 inferences a given model rationally permits. BNs can capture (in)dependencies between sources

139 (e.g. Pilditch et al., 2020; Pilditch et al., 2018), and the influence of perceived reliability on

140 belief revision (e.g. Madsen et al., 2018, 2020), both critical features of misinformation

141 retraction scenarios. Following these studies, which explore the effects of contradictions when

142 considering issues of dependence, we manipulate the retraction. In our case, the source of the 


\section{THE RATIONAL CONTINUED INFLUENCE OF MISINFORMATION}

143 retraction is either the original misinformer or a different source retracts the misinformation 144 statement made by another source.

145 Bayesian normative frameworks facilitate the integration of people's subjective

146 perception of the strength of evidence, their prior beliefs in hypotheses, and their perception of

147 dependency and reliability. Bayesian approaches have been used to explain reasoning biases or 148 errors from a rational perspective, including arguments from ignorance (Hahn, Oaksford, \& 149 Bayindir, 2005; Oaksford \& Hahn, 2004), ad hominem (Harris et al., 2012; Oaksford \& Hahn, 150 2012), slippery slope (Corner et al., 2011), and circular arguments (Hahn, Oaksford, \& Corner, 151 2005). Bayesian networks have also been successfully applied to responses which appear to 152 violate optimal responding, and involve contrary updating, such as belief polarization (Cook \& 153 Lewandowsky, 2016; Jern et al., 2014). By developing process-oriented models, such as

154 Bayesian models, researchers can uncover causal mechanisms, and thereby better test 155 interventions to prevent undesirable outcomes (e.g. the persistence of misinformation after a 156 correction).

157 Exploring the CIE through a formal reasoning model, we find an alternative explanation 158 that does not entail irrationality or bias. In line with model predictions, as explained below, we 159 show that belief in the hypothesis (i.e. misinformation) should remain above prior levels. Instead, 160 the reliabilities of sources that provide contradictory information are (appropriately) penalized 161 whereby people perceive the second (retracting) reporter as less reliable than the first. Temporal 162 dependence influences the effect when incorporated within the formal reasoning model.

163 Correcting is often done by a source that is, in some way, linked with the source of

164 misinformation (e.g. a second reporter working at the same network as the first). The correction 165 must not only be considered a function of direct evaluation of the hypothesis in question but also 


\section{THE RATIONAL CONTINUED INFLUENCE OF MISINFORMATION}

166 of the (in)accuracy of the reports (i.e. the second report may be erroneous not only because of

167 independent error but also the influence of the preceding report). The model, therefore,

168 highlights a significant conceptual limitation to the traditional framing of CIE, which is silent on

169 the dependency between misinformative and retracting reports. The crucial assumption here is

170 that a correcting source is, ceteris paribus, less likely to be providing a truthful testimony when

171 they are following (and contradicting) the report of another source, than when they are the first

172 source providing an independent testimony. Examples of this inequality would be, for instance,

173 concerns over cover-ups or attempts to control narratives, such as to divert blowback/blame

174 arising from the original report. Lewandowsky et al. (2012) have acknowledged that the

175 dismissal of retractions to misinformation could represent rational integration of prior biases with

176 new information and that in principle, it is possible to instantiate the CIE within a BN. To date,

177 however, the CIE has not been realized within a BN framework.

178 Contrary to the standard interpretation of CIE, we demonstrate that there are reasonable 179 grounds under which people should maintain the misinformation, despite the provision of a

180 correction; thus, the effect does not require deviation-based explanatory theories.

181 Conceptualizing the CIE in this manner also has implications for the kinds of interventions that 182 are likely to be effective at reducing reliance on misinformation.

\section{$183 \quad$ 1.2. Source Reliability}

184 Source reliability is essential for evaluating the evidentiary value of testimony. The quality of 185 the source of information is critical to evaluating the suggested content as - for example, if the 186 source lies (is untrustworthy) or is mistaken (is inexpert), it may be entirely reasonable to 187 disregard the suggested content. Although initially demonstrated normatively (e.g. Bovens \& 188 Hartmann, 2003; Hahn et al., 2009), empirical studies suggest that people incorporate 


\section{THE RATIONAL CONTINUED INFLUENCE OF MISINFORMATION}

189 subjectively perceived source credibility into evaluations of testimony (Harris \& Hahn, 2009;

190 Harris et al., 2016; Madsen, 2016). Indeed, adjusting a source's reliability is prudent if new

191 information, additional contradictory or corroborative reports, or insight into the relationship

192 between sources becomes available (Madsen et al., 2020).

193 We consider the CIE to be a case of contradicting testimonies, such that, the corrector's

194 statement contradicts the misinformer's statement, and argue that the correcting source's

195 reliability suffers as a consequence. Several studies support this interpretation and show that the

196 CIE may occur because some people do not believe the retraction (Guillory \& Geraci, 2010,

197 2013; Ithisuphalap et al., 2020; O'Rear \& Radvansky, 2020), demonstrating that source

198 reliability is a critical component of processing retractions to misinformation. As the CIE

199 involves a temporal dependence (the contradicting testimony follows the original, incorrect

200 testimony), there is an additional reason for including source reliability within the scope of the

201 study: the two sources differ in the information they have available (when reporting, the

202 correcting source is often aware of the preceding, incorrect source's statement, but not vice-

203 versa), and this may influence judgments of reliability. In summary, given the CIE involves

204 contradicting testimonies from sources with potentially different access and motivations for

205 producing said testimony, there is a need for formalization, detailed in the section that follows.

206 1.3. A Bayesian Approach to Continued Influence of Misinformation

207 As mentioned, past CIE research has not provided a normative account of how people

208 should process retractions of misinformation. Bayes' theorem gives a normative belief revision

209 model by integrating people's subjective prior degrees of belief with the likelihood ratio to

210 estimate the posterior degree of belief and expresses how a rational agent should revise their

211 belief in a hypothesis $\mathrm{H}$ when faced with new evidence $\mathrm{E}$. The probability $\mathrm{P}(\mathrm{H} \mid \mathrm{E})$ represents the 


\section{THE RATIONAL CONTINUED INFLUENCE OF MISINFORMATION}

212 revised (posterior) degree of belief in the hypothesis $\mathrm{H}$. The revised belief is a function of the

213 prior belief $\mathrm{P}(\mathrm{H})$ and the conditional probability of observing the evidence $\mathrm{E}$ given $\mathrm{H}$ is true.

214 Bayesian approaches to belief revision have been popular in research on argumentation (Hahn \&

215 Oaksford, 2006, 2007), and reasoning (Hayes et al., 2019; Oaksford \& Chater, 2007), as well as

216 other areas of cognition (Chater et al., 2010).

217 The BN framework (Pearl, 1988) is apt for capturing the difficulties of dependencies, and 218 reasoning under uncertainty, that is integral to updating inferences in CIE. Bayesian networks are 219 probabilistic graphical models which represent the relations between items of evidence and 220 possible hypotheses allowing one to draw inferences about specific hypotheses based on 221 observed evidence. The graph consists of a set of nodes representing variables of interest (i.e.

222 hypotheses, evidence, reliability) and a set of directed links representing the probabilistic

223 relations between variables, and in particular, the conditional dependencies. The quantitative

224 component of BNs consists of conditional probability distributions for each variable in the graph.

225 Bayesian networks, therefore, provide the means to test causal models of scenarios - including 226 models of source reliability - and compare intuitive inferences of lay reasoners to a normative 227 standard (Lagnado et al., 2013). The BN framework, therefore, offers a method for formalizing 228 the temporal dependency between misinformative and retracting reports and the impact that this 229 contradiction has on misinforming and retracting sources.

\section{1.4. The Present Study}

231 In order to test the foundational assumptions of CIE formally, we constructed two BN

232 scenarios; in the first scenario, the contradicting report (retraction) comes from the same source

233 whereas, in the second scenario, the contradicting report comes from a second source. Figures 1

234 and 2 (below) show example BN models for the same and different source retraction conditions, 


\section{THE RATIONAL CONTINUED INFLUENCE OF MISINFORMATION}

235 respectively, and illustrate the assumptions of the temporal dependence between first and second

236 reports and the impact on source reliability. Each model consists of a hypothesis node (i.e. the

237 subject of the misinformation report), reporter nodes and reliability node(s). Figures 1 and 2

238 encapsulate the three stages involved in the CIE: the first (baseline) stage reflects the situation

239 when there are no observations; at stage two a single piece of evidence (the misinformation) is

240 available, and at stage three the contradictory (retraction) report is available. Referring to Figures

2411 and 2, the values shown in stage one represent the prior probabilities elicited from participants

242 before reading any reports (see Method section for further details). Each scenario includes

243 unidirectional links between sources to represent the dependency between timepoints or reports.

244 This link represents the assumption that an individual is aware of their previous statements or

245 that, in general, people are aware of existing statements in the 'world' (i.e. a retraction usually

246 requires an awareness of the retracted statement). At stage two, the 'misinforming' reporter node

247 is fixed to 'true' to reflect the positive report submitted by the reporter. The hypothesis node

248 (denoted by $\mathrm{H}$ ) value increases from stage 1 to stage 2, reflecting an increase in belief in the

249 hypothesis (misinformation) after receiving a positive report. At stage three, the hypothesis and

250 reliability nodes update when there is second a contradictory (retraction) report. In both the same

251 and different source cases, belief in the hypothesis decreases relative to stage two but does not

252 return the level observed in stage one, indicating a CIE. There are corresponding updates to the

253 source reliabilities. In Figure 1, the same source reports the misinformation and retraction and

254 source reliability increases from stage one to two but decreases in stage three after the retraction.

255 Figure 2 shows that when a different source retracts the misinformation, the reliabilities of both

256 the first and second reporters decrease from stage two to three. 


\section{THE RATIONAL CONTINUED INFLUENCE OF MISINFORMATION}

Using a BN formalism, we examine the impact of source reliability on the estimated

258 probability of the reported event being 'misinformation' in each scenario - given the statements

259 provided by those sources. For example, this conditional influence of reliability should mean that

260 a source perceived as reliable will be more effective in persuading an individual of their

261 (misleading, or correcting) statement (i.e., misinformation is less likely to be provided,

262 conditional on that source being reliable). Crucially, along with the inclusion of reliability, we

263 also capture a reasonable assumption of dependence between sources (within a single source, or

264 between different sources) as correction temporally follows misinformation. Taken together with

265 the consideration of reliability, we argue this yields a rational explanation for CIE. The model

266 can consequently capture key differences between conditions. For example, it captures the clear

267 difference in how reliability is updated (and belief in misinformation is also updated) when a

268 single source contradicts itself, vs when a different source provides the contradiction. In the

269 single-source scenario, the reliability of the source is penalized more heavily than in the two-

270 source scenario because of the internal contradiction. Lastly, we elicit key parameters from

271 participants themselves, such that we fit each model to participant's assumptions. As a result of

272 this, we can investigate the consistency of participant responses relative to the predictions of

273 their own, fitted models. Following the CIE paradigm, participants in the present study read a set

274 of brief news reports and complete a comprehension test. Crucially, we varied whether or not a

275 sentence that appeared towards the end of the report retracted information provided earlier, and

276 whether the retracting source was the same or different to the misinforming source.

277 In this paper, we examine four hypotheses. We take pains to note that we do not base

278 these predictions on the parameterizations of Figures 1 and 2, as these are solely illustrative

279 examples of the more general structural (and ordinal parameter) relations that we note can lead to 


\section{THE RATIONAL CONTINUED INFLUENCE OF MISINFORMATION}

280 a rationally predicted CIE. The model is used to delineate several underpinning assumptions and

281 effects that we outline below:

$282 \mathrm{H} 1$ : We predicted higher endorsement of misinformation probes in the retraction condition than

283 in the control condition in which there was no retraction (i.e. there is no retraction of the initial

284 report).

285 H2: We predict that the conditional probability measures provided by participants (which assess 286 participant's interpretation of the relationship between a source's likelihood of a statement being 287 in error, given their reliability and possible contradiction of previous statements) will yield a 288 predicted CIE when using these measures to parameterize the Bayesian network model.

289 H3: In line with model predictions, participants will penalize source reliability when there is a

290 contradiction. The perceived reliability of the retracting source (at stage three) will decrease

291 relative to misinforming source (at stage two), as shown in Figures 1 and 2. The same vs

292 different source manipulation is exploratory, and there is no directional prediction for the impact 293 on reliability.

294 H4: We expect to elicit the CIE in terms of the posterior probability measure such that 295 participants will retain belief in the hypothesis (i.e. the misinformation) despite a retraction. 
THE RATIONAL CONTINUED INFLUENCE OF MISINFORMATION

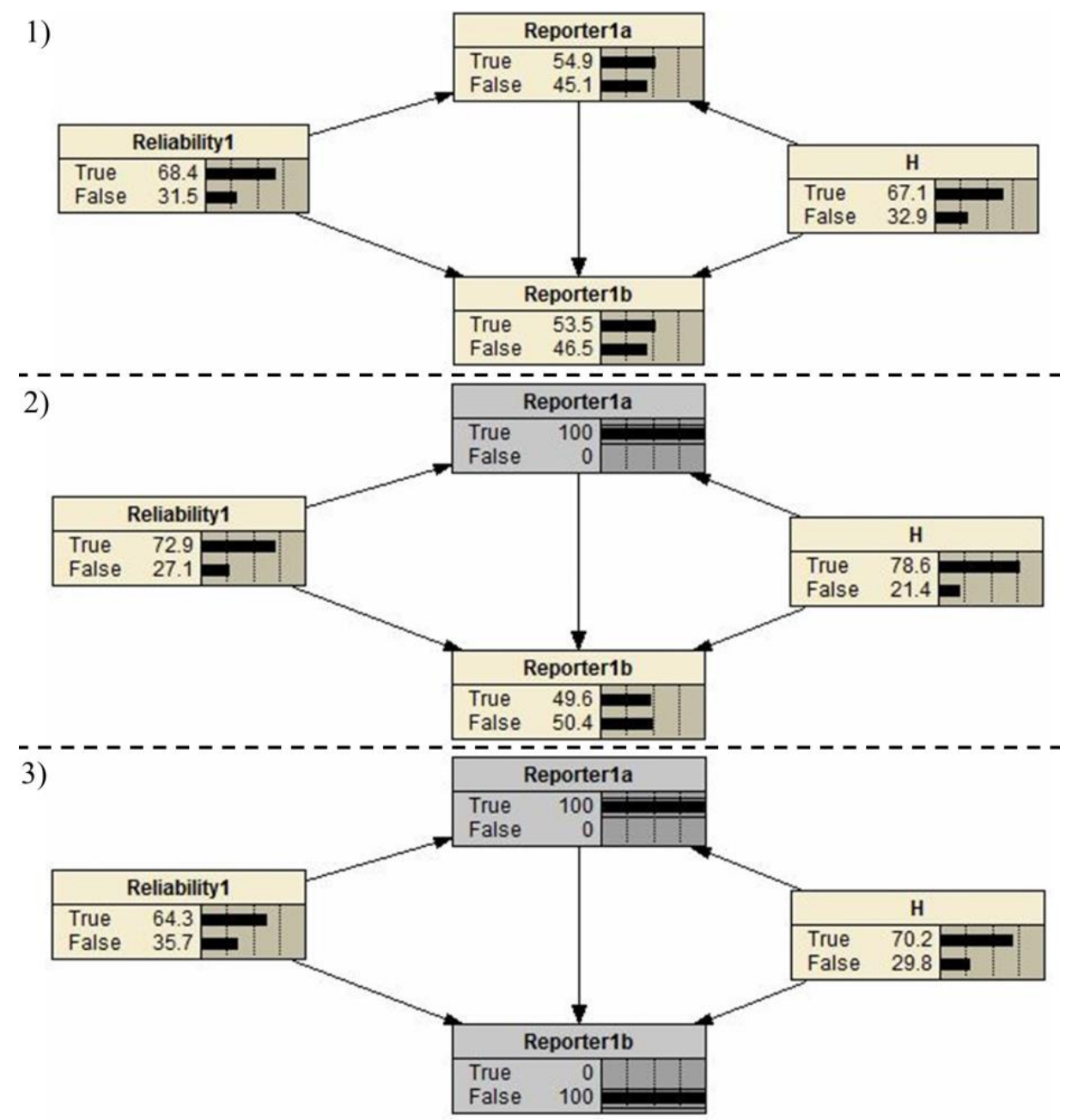

Fig $1 \mathrm{BN}$ model for the retraction (same source) condition across three different time points, where $\mathrm{H}$ represents the belief in the hypothesis in question (misinformation), which is informed by the misinformer (represented as Reporter1a) and later this same source as a correction (Reporter1b). Given this is the same source at two points in time, the reliability of the source (Reliability1) connects to both instances of the reporting source. 1) Baseline (no observation) stage, 2) Single positive (first) report stage (i.e. control 


\section{THE RATIONAL CONTINUED INFLUENCE OF MISINFORMATION}

condition) -misinformation stated, and 3) Final (retraction) state given a second, correcting report from the same reporter.2 Figure created using the AgenaRisk Bayesian Network software (AgenaRisk, 2019).

$2 \mathrm{BN}$ model parameters taken from the mean estimates across the retraction same condition for the police officer scenario. 
THE RATIONAL CONTINUED INFLUENCE OF MISINFORMATION
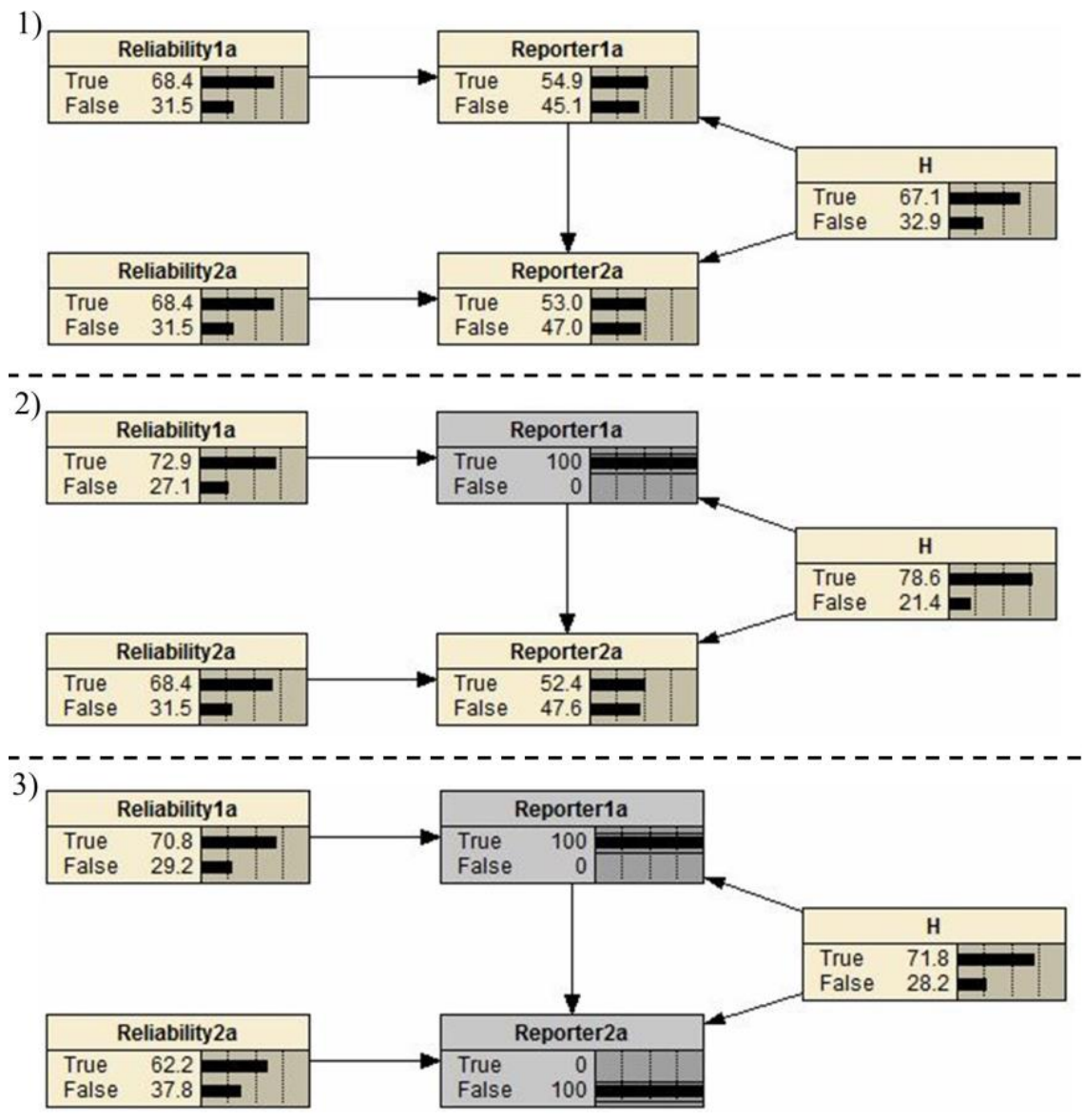

Fig 2 BN model for the retraction (different source) condition across three different time points. $\mathrm{H}$ represents the belief in the hypothesis in question (misinformation), which is informed by the misinformer (represented as Reporter1a) and later a separate source as a corrector (Reporter 2a). Given this, the two sources have their reliability specified (Reliability 1a and 2a, respectively). 1) Baseline (no observation) stage, 2) Single positive (first) report stage (i.e. control condition) - misinformation stated, and 3) Final (retraction) state given a second, correcting report from a separate reporter3.

$3 \mathrm{BN}$ model parameters taken from the mean estimates across the retraction different condition for the police officer scenario. 
THE RATIONAL CONTINUED INFLUENCE OF MISINFORMATION

\section{Method}

\subsection{Participants}

We aimed to recruit 105 participants $(\mathrm{N}=35$ per condition). We based our estimate on the principle that formal statistics sources suggest that Central Limit Theorem tells us that the sampling distributions of the means will be approximately normal, even if the underlying data distributions are non-normal when the sample size is larger than 30 (Field, 2013). There was no prior work to inform an effect-size based power calculation as this was a novel design. In total, 101 participants from Prolific Academic https://www.prolific.co/ completed the experiment. There was a mean age of $31.57(S D=9.6)$, and there were 71 females and 30 males. Participants were paid $£ 1.50(\sim \$ 1.97)$ for their time (Median $=12.87$ minutes, $S D=5.78)$.

\subsection{Materials, Design and Procedure}

To replicate CIE, we used materials adapted from past research (Gordon et al., 2017;

Johnson \& Seifert, 1994). We opted for shorter scenarios than those typically used in CIE studies to keep the study duration to a minimum since participants answered an extensive set of conditional probability questions (see Supplementary Materials https://osf.io/6yq47). It was also necessary to ensure that the non-critical details in the scenario were independent of the hypothesis (misinformation) and the evidence (retraction), to model the participants' responses. We selected four scenarios for the main study, that produced the largest baseline CIE (i.e. the difference between retraction and control conditions) from a set of eight pilot scenarios $(N=70)$.

In the main study, participants read four scenarios (motorcycle accident/police officer, medical controversy/independent reviewer, music festival/local journalist, and explosion/police spokesperson) consisting of six sequentially presented sentences (see Supplementary Materials).

The materials provided minimal information about the source of the misinformation and 


\section{THE RATIONAL CONTINUED INFLUENCE OF MISINFORMATION}

24 retraction, to better control for differences in perceived source reliability. In this sense, the

25 inferences participants make about reliability should be based on the contradiction, and the

26 source's profession (i.e. police officer, local journalist, independent reviewer, police

27 spokesperson).

We assessed the effect of retracting information between groups (Control, Retraction -

29 Same Source, Retraction - Different Source). We randomly assigned participants to a condition

30 and randomized the presentation order of the scenarios across participants. Table 1 shows that in

31 each scenario, sentence 2 differed between control and retraction conditions for each event. In

32 retraction conditions, sentence 2 contained (mis)information. Whereas in the control condition,

33 sentence 2 contained incidental information to provide a baseline for the misinformation

34 endorsement test. The key sentence (sentence 5) was identical in all conditions. Given exposure

35 to sentence 2 , sentence 5 did or did not correct previous information. The source of the

36 (mis)information (sentence 2) and retraction (sentence 5) were either from the same source or a

37 different source, in the retraction conditions.

38 Before reading the scenarios, participants provided prior estimates for the reliability of

39 the sources of misinformation that would appear in the subsequent reports on a scale of 0

40 (Extremely unlikely) to 100 (Extremely likely). They then provided six conditional probability

41 estimates per report for each of the two sources (i.e. the misinformer and the retractor). Eliciting

42 conditional probability estimates in this way is necessary because there is no general normative

43 function that captures the dependency relationship between the misinformer and the retractor.

44 Participants provided their probability estimates (on the same scale as above) for the same and

45 different source conditions and thus capturing the specific assumptions regarding the nature of

46 the dependency relationship on the individual level. 


\section{THE RATIONAL CONTINUED INFLUENCE OF MISINFORMATION}

1. If a police officer is reliable, how likely are they to make an erroneous statement in reporting about a road accident, if that same police officer is contradicting their own earlier statement?

2. If a police officer is reliable, how likely are they to make an erroneous statement in reporting about a road accident, if that same police officer is contradicting an earlier statement from another police officer?

Responses to the above questions illustrate one aspect of the dependency relationship: the perceived likelihood of a source providing an erroneous report (i.e. the misinformation) given that they are reliable, before learning about the specifics of the event. Participants provided six conditional probability estimates per event scenario (24 in total), all on 0-100 sliders ( 0 and 100 denote the same as in the above). Due to the elicitation of conditional probabilities, no free parameters were requiring posthoc fitting.

The modelling process generated a Condition (3) x Scenario (4) matrix, creating 12 "group" models. Participants provided three types of estimates; participants supplied the first two types of estimates before reading the reports and supplied the third type of estimate after reading the report. First, participants provided estimates for the reliability of the sources of misinformation that would appear in the subsequent reports (e.g. police officer), which we call reliability priors (e.g. How likely are police officers to be reliable in their reporting?). Second, participants estimated the likelihood of the sources making an erroneous statement about the reported event (e.g. road accident) conditional on the source being reliable or not (e.g. If a police officer is reliable, how likely are they to make an erroneous statement in reporting about a road accident?), and conditional on whether the source was contradicting/corroborating their own or another source's statement. We call these estimates conditional probability estimates. These 


\section{THE RATIONAL CONTINUED INFLUENCE OF MISINFORMATION}

70 allow for a full range of possible assumptions regarding the relationship between the

71 misinforming and retracting reports (from no influence, to complete dependence). Finally,

72 participants estimated the probability of the focal hypothesis and the reliability of the source in

73 each scenario.

74 We created each model using the elicited responses for each estimate, using as many of

75 the responses of possible. All three conditions could use the reliability priors from all

76 participants, along with the conditional probabilities for Reporter 1, as these were similar across

77 conditions. However, the conditional probabilities for Reporter 2 were condition-specific (i.e. the

78 Retraction Same Source condition could only use conditionals elicited from that condition - see

79 question 1 above).

80 Lastly, as eliciting participants' "prior" probability estimates for the focal hypothesis in

81 each scenario (i.e. an estimate of how likely the reported event is to be accurate, in each

82 scenario) beforehand were likely to interfere with CIE (via the premature introduction of the

83 misinformation), we reverse-engineered the priors from the control condition posterior estimates.

84 As the control condition had a single positive Reporter 1 observation (rather than multiple

85 contradicting observations), a prior probability could be calculated via Bayes Theorem using the

86 known likelihood (i.e. Reporter 1 conditionalized parameters) and provided posterior estimates.

87 For example, given the posterior (P ( Hypothesis | Report) for the journalist scenario was

$8877.58 \%$, and the elicited conditional probabilities for the journalist reporter were a probability of

89 the journalist being correct if reliable (P ( Report | Hypothesis, Reliable)) of $68.94 \%$ and correct

90 if unreliable (P (Report | Hypothesis, $\neg$ Reliable)) of 31.06\% (with these probabilities reflected

91 for the chance of error), conditional on a probability of being reliable (P (Reliable)) of 54.1\%,

92 then dividing the above posterior by the conditionalized reporter likelihood results in a prior 


\section{THE RATIONAL CONTINUED INFLUENCE OF MISINFORMATION}

93 (P(Hypothesis)) of 75.6\%. All parameters, except the generated prior, were directly elicited from

94 participants and fed into the model at the group level. This "prior" could then be implanted in the

95 retraction condition models, further ensuring that the models did not have any free parameters.

96 Responses to a set of misinformation probes that followed each scenario measured the

97 continued influence of misinformation (see Table 1). Participants rated each probe on a 7-point

98 scale from 'strongly disagree' to 'strongly agree'. In line with previous CIE methods, probes

99 referred to the critical information (sentence 5). Higher levels of misinformation probe

100 endorsement captured the extent to which participants integrated the misinformation (sentence 2

101 in the retraction conditions) into their understanding of the news report.

102 After rating the probes, participants provided posterior probabilities on a similar scale

103 used for prior beliefs. For example, in the scenario in Table 1, participants were asked: 1) Given

104 everything you know so far about the incident in question, how likely do you think it is that

105 the accident occurred because the driver was intoxicated/travelling over the speed limit? 2)

106 Given everything you know so far about the incident in question, how likely to do you think it is

107 that the police officer is reliable in their reporting? Participants who received a retraction from a

108 different source as the misinformation provided an additional estimate for the reliability of the

109 second reporter. 
THE RATIONAL CONTINUED INFLUENCE OF MISINFORMATION

Table 1

Example news report scenario and misinformation probes

\begin{tabular}{llcc}
\hline Sentence & Control & $\begin{array}{c}\text { Retraction } \\
(\text { Same Source })\end{array}$ & $\begin{array}{c}\text { Retraction } \\
\text { (Different Source) }\end{array}$ \\
\hline
\end{tabular}

\section{Example News Report}

Sentence 1 A motorcyclist died yesterday after being knocked off his bike by a car.

$\begin{array}{llll}\text { Sentence } 2 & \begin{array}{l}\text { Officer Jones reported } \\ \text { that the driver of the car } \\ \text { had been travelling over } \\ \text { the speed limit. }\end{array} & \begin{array}{l}\text { Officer Jones reported } \\ \text { that the driver of the } \\ \text { car was intoxicated. }\end{array} & \begin{array}{l}\text { Officer Jones reported } \\ \text { that the driver of the } \\ \text { car was intoxicated. }\end{array}\end{array}$

Sentence 3 The accident happened on the A7 north of Carlisle.

Sentence 4 The motorcyclist was 30 years old and had two children.

$\begin{array}{llll}\text { Sentence } 5 & \begin{array}{l}\text { Officer Jones revealed } \\ \text { that the car driver was not } \\ \text { intoxicated. }\end{array} & \begin{array}{l}\text { Officer Jones revealed } \\ \text { that the car driver was } \\ \text { not intoxicated. }\end{array} & \begin{array}{l}\text { Officer Smith revealed } \\ \text { that the car driver was } \\ \text { not intoxicated }\end{array}\end{array}$

Sentence 6 The driver of the car was also injured in the incident.

\section{Example Misinformation Probes}

Question 1 Drink-driving charges should be brought against the driver of the car

Question 2 The driver should be forced to complete a drink-driving awareness course

Question 3 A breathalyzer would have returned a positive result 


\section{THE RATIONAL CONTINUED INFLUENCE OF MISINFORMATION}

\section{Results}

Bayesian analyses were performed with JASP statistical software (JASP Team, 2018) and assumed an uninformed prior. The use of Bayes factors (BFs) additionally allowed us to infer evidence for the null hypothesis, wherein a $\mathrm{BF}_{10}$ of less than one third is considered substantial support for the null (Dienes, 2014).

\subsection{Misinformation Endorsement Ratings}

A Bayesian repeated-measures ANOVA was used to determine the effect of retraction condition and scenario type on mean misinformation endorsement ratings. Strong evidence was found for the main effect of condition, $\mathrm{BF}$ Inclusion $4=1.917 * 1012$, and scenario, $\mathrm{BF}$ Inclusion $=5.44 *$ 109 , but no interaction, BFInclusion $=0.122$. The model including just main effects was the strongest fit, $\mathrm{BF} 5=131.26$, and was decisive; overall, $\mathrm{BF}_{10}=2.105 * 1022$. As illustrated in Fig. 3 scenarios differed in misinformation endorsement ratings from one another, and there was a differential influence of condition.

Critically, the effect of condition indicated significantly higher endorsement ratings following the presentation and retraction of misinformation compared to when there was no misinformation presented. This result indicates that a CIE was observed across all scenarios, such that a retraction was insufficient to bring endorsement ratings back to baseline.

A Bayesian repeated-measures ANOVA was also used to establish whether there was an effect of scenario order (i.e. whether a participant read the scenario first, second, third or fourth) on misinformation endorsement ratings. This found no effect of scenario order, BFInclusion $=$

$4 \mathrm{BF}$ Inclusion reflects the change in odds from the sum of the prior probabilities of models that include the effect, to the sum of posterior probabilities of models including the effect. $5 \mathrm{BF}$ reflects the change from prior to posterior odds for the given model. 


\section{THE RATIONAL CONTINUED INFLUENCE OF MISINFORMATION}

210.435 , and strong evidence for a null effect of its interaction with scenario (type), BFInclusion $=$

22 0.034. There was, however, strong evidence for the main effect of scenario, BFInclusion $=5.46 *$

23109 , with the model including only this main effect yielding the strongest fit, $\mathrm{BFM}=6.132$, and

24 decisive overall, $\mathrm{BF}_{10}=7.54 * 109$. 


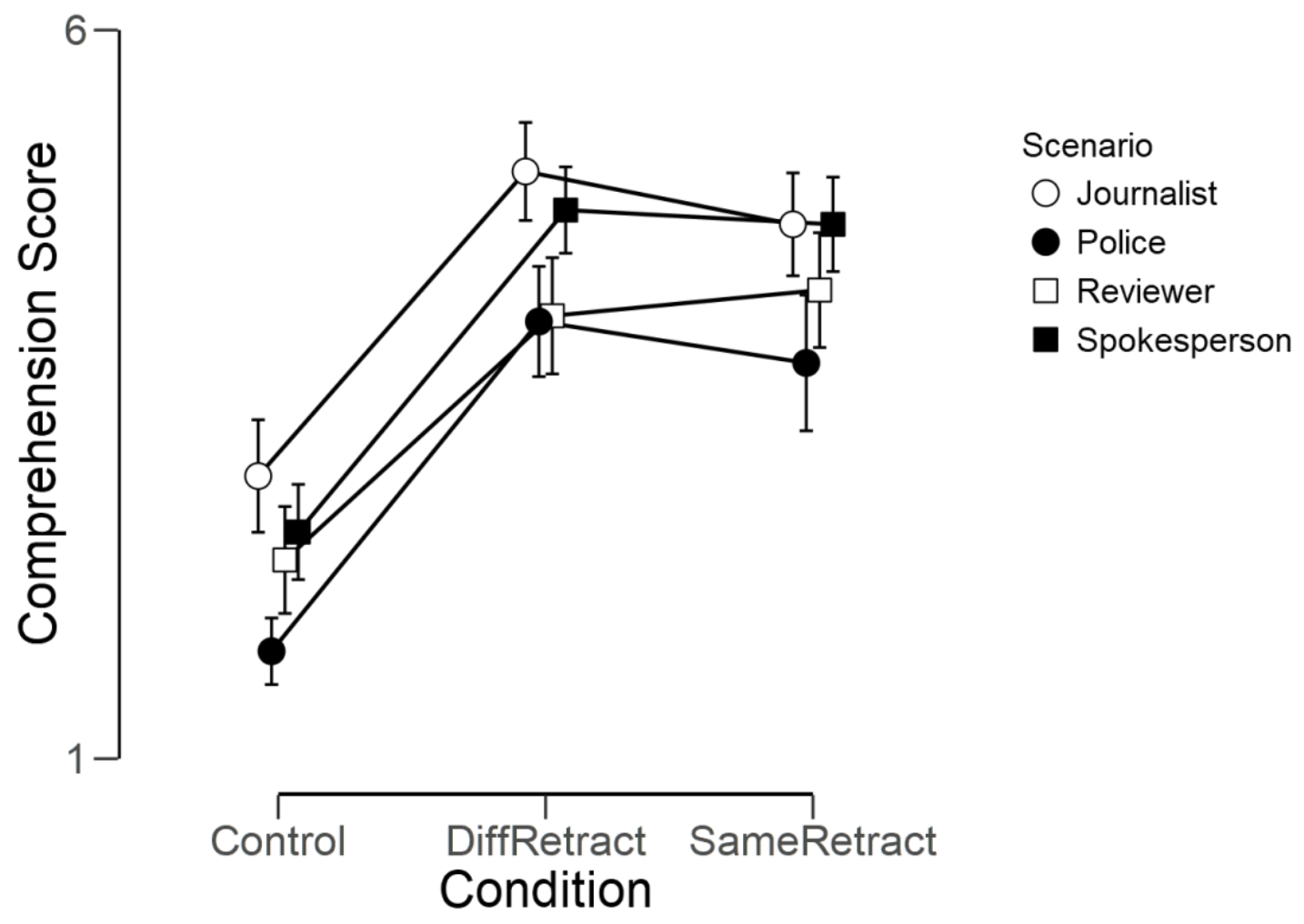

Fig 1 Mean misinformation endorsement ratings, split by scenario (line) and condition (horizontal axis). Error bars reflect $95 \%$ CI. The scale ranged from $1=$ strongly disagree to $7=$ strongly agree. Misinformation probes were more strongly endorsed in the retraction conditions than the control condition. A retraction was insufficient to bring endorsement ratings to baseline levels. Note that points have been offset on the x-axis to improve legibility. 


\section{THE RATIONAL CONTINUED INFLUENCE OF MISINFORMATION}

\subsection{Bayesian Model Fits}

Using the conditional probabilities and priors elicited from participants, group means of

3 these estimates were used to parameterize two group-condition models for each scenario. The

4 conditional probabilities and priors for each first reporter and reliability node were fitted based on

5 all participants, with two notable exceptions. First, conditional probabilities for the second reporter

6 were based solely on estimates from the condition of relevance (i.e. we only used estimates from

7 the retraction (different source) condition to parameterize the entailed different second reporter in

8 that condition). Secondly, we reverse-engineered prior probabilities for each hypothesis (via Bayes

9 Theorem) using the posteriors provided by the control condition. More precisely, taking the control

10 condition BN model, the posterior for the hypothesis was fitted, given the single positive report.

11 Retracting the observation could reveal the approximate prior (absent observations) for that

12 hypothesis. This "prior" was fitted into the models for the two retraction conditions.

13 Figures 1 and 2 illustrate models for each experimental condition of the police officer

14 scenario, fitted from participant data according to the protocol outlined above. Several significant

15 trends are noticeable: Firstly, as expected, given a single positive reporter (stage 2), belief in the

16 hypothesis $(\mathrm{H})$ increases, and the predicted likelihood of corroboration from the second report

17 increases. However, when the second, contradicting report is observed (stage 3), the belief in the

18 hypothesis $(\mathrm{H})$ does not return to prior (stage 1) levels. Instead, the reliability of sources decreases

19 given the contradiction, this decrease is most influential in the second reporter (different condition)

20 but is also substantial when the same reporter contradicts themselves (Fig. 2, stage 2 to stage 3 ).

21 Critically, the reason for this effect (retention of belief in $\mathrm{H}$, but the reduction in

22 perceived reliability) is due to the capturing of the temporal dependence from first to the second

23 report. Put another way; the models capture the intuition that the second report is made with an 


\section{THE RATIONAL CONTINUED INFLUENCE OF MISINFORMATION}

24 awareness of the first report (whether internally in the case of the same reporter condition or via

25 general narrative in the different reporter condition). The elicited conditional probabilities from

26 participants then capture the manner and strength of this influence.

3.3. Participant Estimates

Returning to participant data, we again use Bayesian repeated-measures ANOVA to examine whether probability estimates correspond to the BN model predictions (and thus map onto a CIE) or corroborate the misinformation endorsement ratings (and indicate an absence of

31 CIE - against fitted normative prescription).

\section{3.3.1. Hypothesis}

Turning first to posterior estimates of belief in the hypothesis, we find main effects of

34 condition, $\mathrm{BFInclusion}=3.328 * 109$, and scenario, $\mathrm{BF}$ Inclusion $=41812.52$, but no interaction,

35 BFInclusion $=0.467$. The model consisting of the main effects along was the strongest fit, $\mathrm{BF}=$

3634.27 , and enjoyed strong support overall, $\mathrm{BF}_{10}=2.247 * 10_{14}$. As Fig. 4 illustrates, these effects

37 corroborate misinformation endorsement ratings; wherein there is the retention of belief in

38 misinformation despite its retraction. Crucially, this shows that participants generally deviate

39 from the prescribed CIE entailed by the BN models, decreasing belief in the hypothesis below

40 the control condition (and prior), given the retraction.

41 We again checked for order effects for posterior estimates of belief in the hypothesis

42 across scenarios, finding strong evidence for a null effect of presentation order, BFInclusion $=0.028$,

43 and its interaction with scenario type, BFInclusion $=0.02$. There was, however, the main effect of

44 scenario type, BFInclusion $=5354.61$, with the model including only scenario type yielding the

45 strongest fit, $\mathrm{BFM}=94.26$ and being decisive overall, $\mathrm{BF}_{10}=7963.11$. 


\section{THE RATIONAL CONTINUED INFLUENCE OF MISINFORMATION}

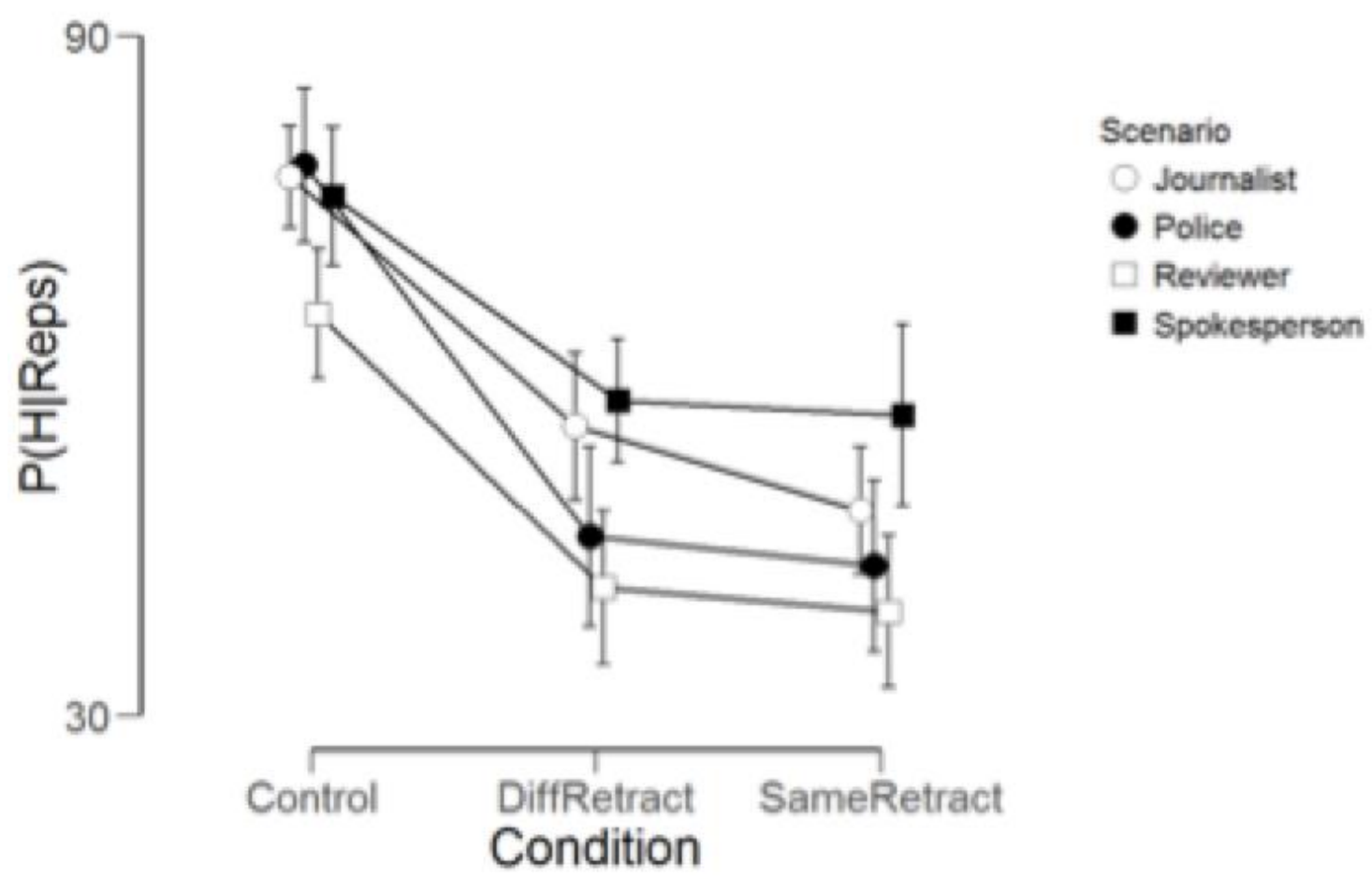

Fig 2 Posterior estimates of belief in the hypothesis $(\mathrm{H})$, given all reports, split by scenario (line) and condition (horizontal axis). Error bars reflect 95\% CI. Note that points have been offset on the $\mathrm{x}$-axis to improve legibility. 


\section{THE RATIONAL CONTINUED INFLUENCE OF MISINFORMATION}

\subsubsection{Individual-Level Analysis}

We performed an exploratory analysis to examine individual differences in participants' model predictions, posterior estimates of belief in the hypothesis, and their misinformation

51 endorsement ratings. We first generated individual model fits for each participant and scenario.

52 These individual fits were based on each participant's prior estimate of reliability for the source

53 of the reports, conditional probabilities for sources, and the prior probability of the

54 (misinformed) report being true, which was reversed engineered from the control group mean for

55 that scenario (as such a prior could not be elicited from the same participant without

56 undermining the CIE framework premise - see section 2.2). A prediction of CIE was defined as

57 observing a posterior probability for the hypothesis after both reports that remained above the

58 level of the prior. We computed a measure of observed CIE by subtracting the group level prior

59 for the control condition from the final posterior $\mathrm{p}(\mathrm{H} \mid \mathrm{Reps})$, wherein positive values indicate

60 retention of the misinformation despite a retraction. We then computed the proportion of

61 participants whose fitted BN model predicted a CIE and the proportion of participants who

62 exhibited a CIE (i.e. retained belief in the misinformation despite a retraction), separately for

63 each of the four scenarios tested (see Table 2).

64 The first finding of note from is that although around half of participants provided

65 parameter estimates that should lead to the CIE, very few do. We confirmed this by performing

66 Bayesian tests of association using a joint multinomial sampling plan and default priors,

67 separately for each scenario6, to test the null that there was no association between observed and 68 predicted CIE. The journalist scenario produced a $\mathrm{BF}_{01}=3.020$, the reviewer and police officer

6 It was necessary to perform separate analyses for each scenario as the levels of scenario were not independent. 


\section{THE RATIONAL CONTINUED INFLUENCE OF MISINFORMATION}

69 scenarios produced $\mathrm{BF}_{01}=3.618$, and the spokesperson scenario produced a $\mathrm{BF}_{01}=2.233$,

70 indicating evidence for the null was inconclusive.

72 Table 2 Percentage of participants with predicted given their BN model and dichotomized

73 posterior probability judgments

\begin{tabular}{cccccc}
\hline $\begin{array}{c}\text { Predicted } \\
\text { CIE }\end{array}$ & $\begin{array}{c}\text { Observed } \\
\text { CIE }\end{array}$ & Journalist & Police & Reviewer & Spokesperson \\
\hline Yes & No & 39.71 & 38.24 & 47.06 & 32.84 \\
Yes & Yes & 5.88 & 32.35 & 5.88 & 14.93 \\
No & No & 45.59 & 23.53 & 42.65 & 38.81 \\
No & Yes & 8.82 & 5.88 & 4.41 & 13.43 \\
\hline
\end{tabular}

74

75 To corroborate this finding, we also performed a Bayesian regression to examine whether

76 participant's parameter estimates predicted their misinformation endorsement ratings. The model

77 included whether or not a participant's BN model predicted a CIE as a fixed effect and

78 participant as a random effect. The model, including BN model predictions as a fixed effect, was

792.11 more likely than an intercept only model7. Therefore, there was only anecdotal evidence

80 that participants with a BN that predicted a CIE went on also to endorse misinformation.

81 Taken together, we find that although on an individual basis, many participants detailed

82 probabilistic relationships between model components that should produce a CIE, very few

83 participants went on to exhibit one in their probability responses. Furthermore, inclusion in the

84 former category did not predict inclusion in the latter. Finally, there was anecdotal evidence that

85 each participant's parameter estimates predicted their misinformation endorsement ratings.

7 The Bayesian regression was performed using the BayesFactor package in $\mathrm{R}$ using default Cauchy priors. 
THE RATIONAL CONTINUED INFLUENCE OF MISINFORMATION

\section{3.3.3. Reliability}

Turning next to estimates of reliability, we add to the repeated measures ANOVA analysis a within-subject factor of the change in reliability estimates from the prior to posterior.

90 Here we find significant main effects of condition (control > retraction different and same),

91 BFInclusion $>1020$, scenario, BFInclusion $=124.44$, and prior-posterior $($ posterior $<$ prior $)$, BFInclusion $>$

92 1020. Figs 5, 6, and 7 illustrate the significant interaction of condition and prior-posterior,

93 BFInclusion $>1020$, wherein reliability estimates increased in the control condition (Fig. 5; where

94 no contradiction occurs, and in line with the increase observed in Figs 1 and 2, stage 2), but

95 decreased in both retraction conditions (Figs 6 and 7; also, in line with model predictions

96 illustrated in Figs 1 and 2, stage 3). Lastly, we also observed a strong interaction of scenario and

97 prior-posterior, $\mathrm{BF}_{\text {Inclusion }}=75.92$, wherein the spokesperson scenario entailed smaller changes

98 from the prior to the posterior than the 3 remaining scenarios. The model, including the above-

99 supported terms, yielded the strongest fit, $\mathrm{BFM}=484.97$, and was decisive; overall, $\mathrm{BF}_{10}=1.559$

$100 * 1028$.

101 Finally, we note that the retraction condition showed no significant difference in posterior

102 reliability estimates between the two different (first and second) reporters, $\mathrm{BF} 10=0.135$, contrary

103 to model predictions (wherein there should be a more substantial reliability penalty for the

104 second reporter because of the contradiction). 
THE RATIONAL CONTINUED INFLUENCE OF MISINFORMATION

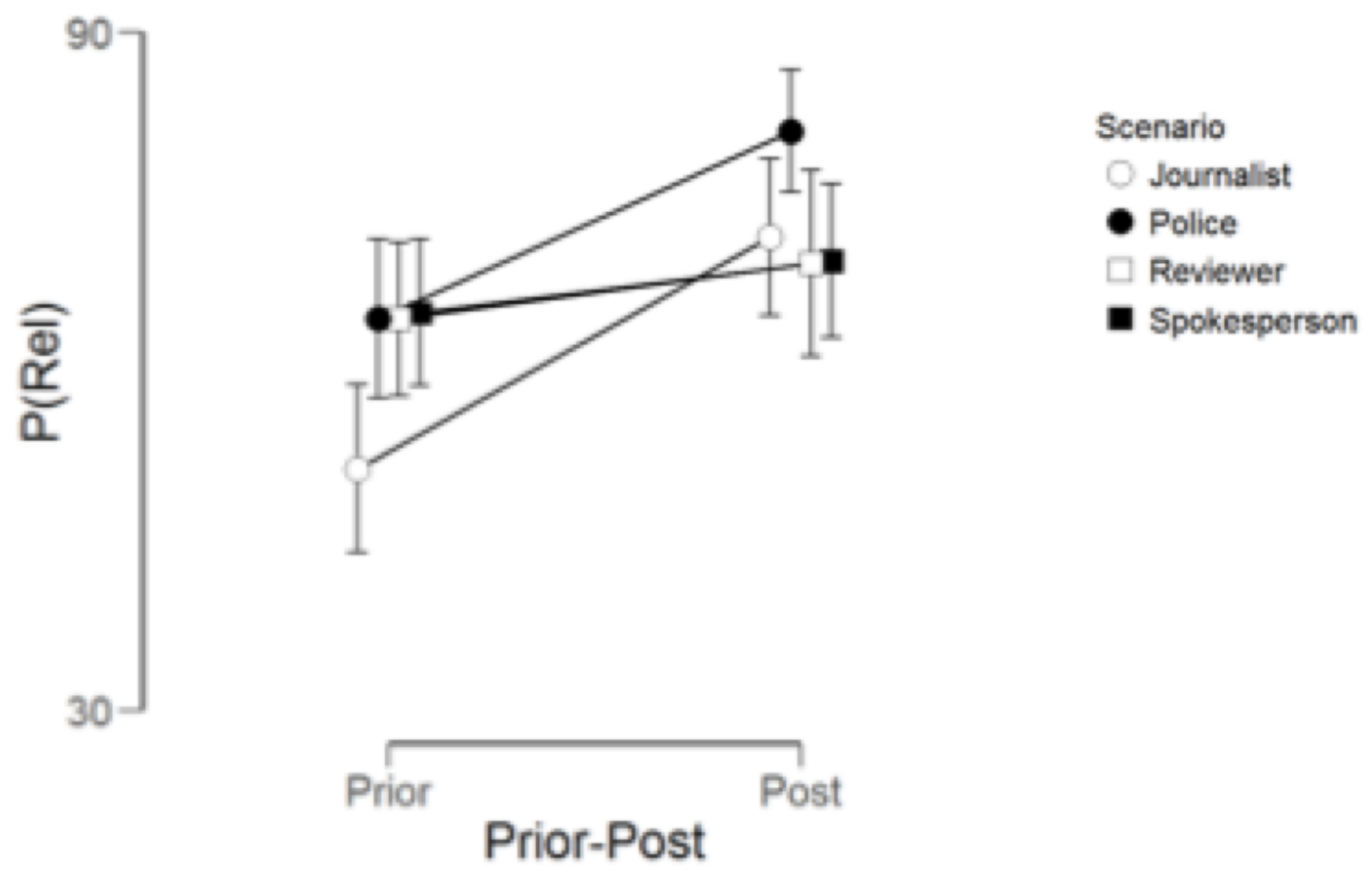

Fig 3 Control condition reliability estimates for reporters from prior to posterior (reports

observed), split by scenario (lines). Error bars reflect 95\% CI. Note that points have been offset on the $\mathrm{x}$-axis to improve legibility. 


\section{THE RATIONAL CONTINUED INFLUENCE OF MISINFORMATION}

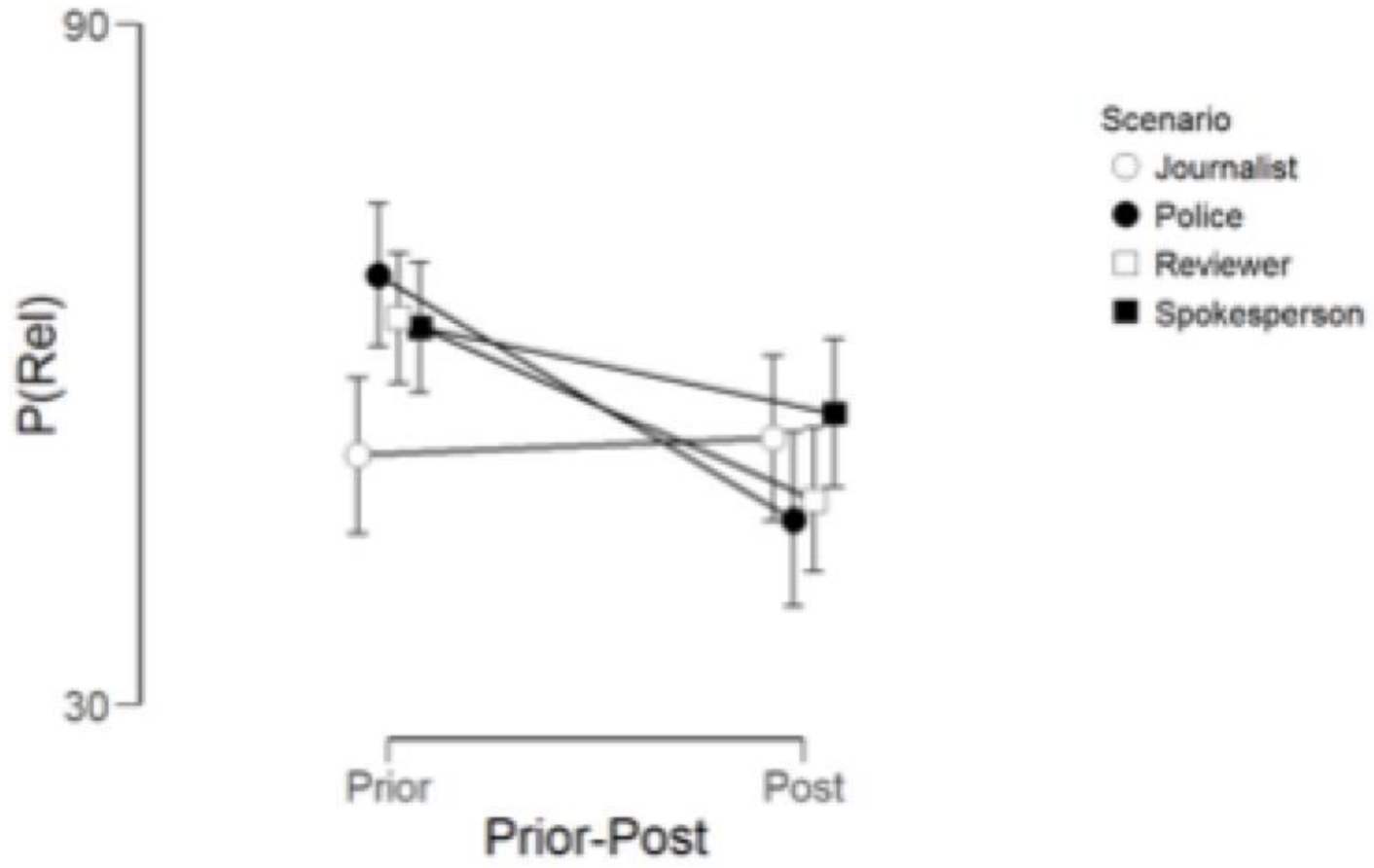

Fig 4 Retraction different condition reliability estimates for reporters from prior to posterior (reports observed), split by scenario (lines). Error bars reflect 95\% CI. 
THE RATIONAL CONTINUED INFLUENCE OF MISINFORMATION

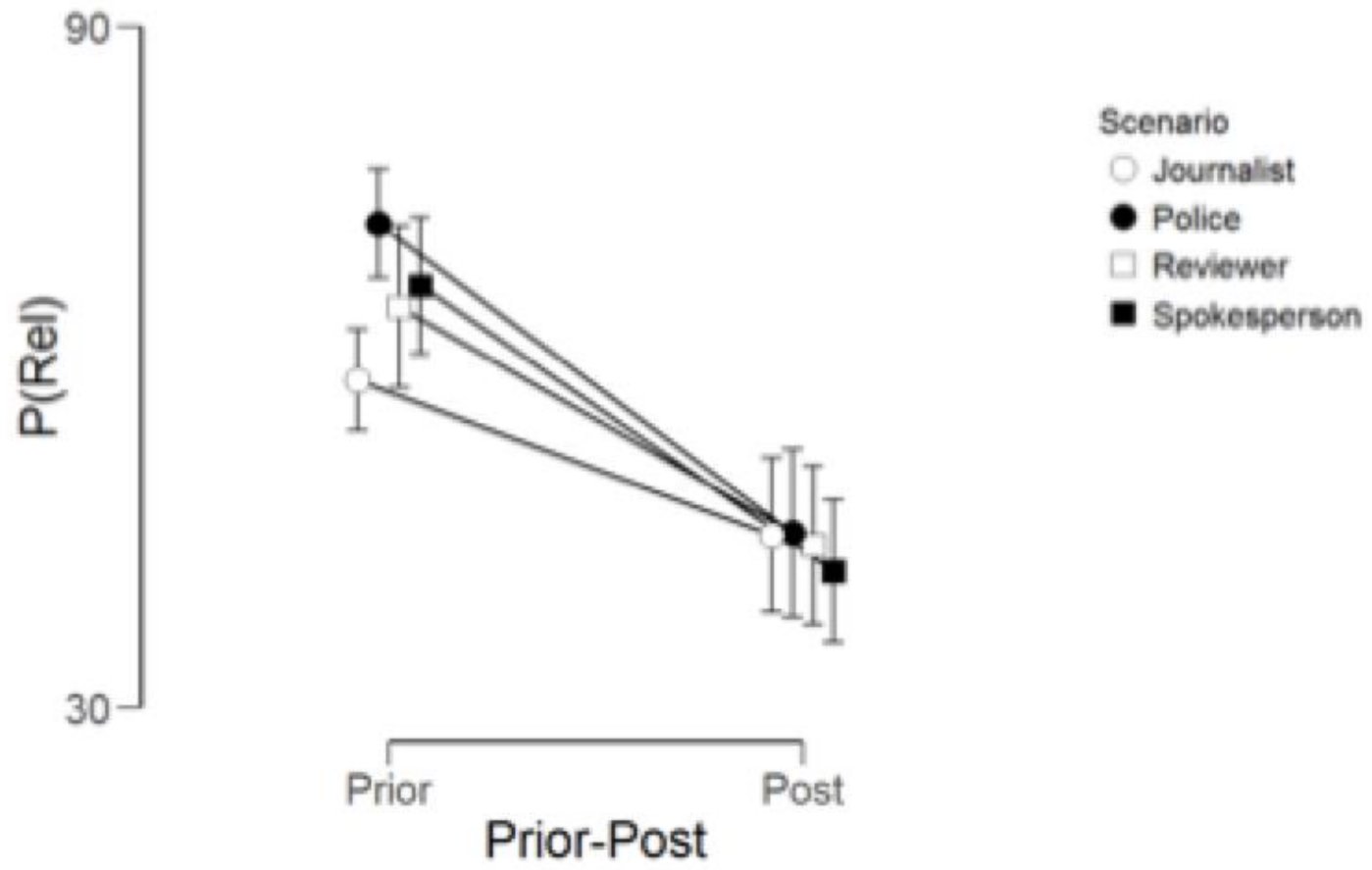

Fig 5 Retraction same condition reliability estimates for reporters from prior to posterior (reports observed), split by scenario (lines). Error bars reflect 95\% CI. 


\section{THE RATIONAL CONTINUED INFLUENCE OF MISINFORMATION}

\section{Discussion}

This paper formalizes the continued influence of misinformation (Johnson \& Seifert, 1994; Lewandowsky et al., 2012) in a Bayesian network model which accounts for the temporal dependency between the misinformation and retraction reports, and its impact on source reliability. When accounting for the temporal dependency between misinformation and its retraction, we find a rational account for the continued influence effect of misinformation. We find that participant's responses broadly fit with the predictions of this account, and show that belief in the hypothesis (i.e. the misinformation) remains above prior level, and instead, participants penalize the reliability of the second reporter (i.e. retraction's source). Participants perceived the second (retracting) reporter as less reliable than the first (misinforming) reporter, irrespective of whether the second reporter was the same or different from the first. However, participant's posterior estimates also decreased below their priors, and against their model predictions. This finding is contrary to standard CIE accounts (that people continue to rely on retracted misinformation when they should not); instead, we show that people do not always continue to rely on misinformation even though they should!

An individual-level analysis of the data revealed that people can, and do, endorse the necessary assumptions for a rational account of the CIE. However, most participants were unable to incorporate these assumptions into their posterior probability judgments or their misinformation endorsement ratings. Put another way many participants did not achieve the complex Bayesian update that the model entails; namely, integrating the conjunction of temporal dependency, and its impact on source reliability, to estimate the strength of the evidence for the retracted misinformative report. Crucially, these findings show that a "rational" CIE is possible when conceptualized in Bayesian terms, even with people's assumptions about the relationships 


\section{THE RATIONAL CONTINUED INFLUENCE OF MISINFORMATION}

24 between the factors in play. This finding shows that the integration of contradictory sources is

25 difficult even when one considers the temporal dependency between the misinformation and

26 retraction.

27 Reliability estimates revealed that participants decreased their estimate for a reporter who

28 contradicts themselves, in line with model predictions. In the different source condition,

29 participants decreased their reliability estimates for the first reporter and increased reliability

30 estimates of the second reporter (both correct according to the model). Interestingly, the second

31 reporter was considered more reliable than the first in the police officer and independent

32 reviewer scenarios, but less reliable than the first in the journalist and police spokesperson

33 scenarios. Descriptively, this discrepancy in reliability estimates demonstrates participant's

34 sensitivity to the different types of sources and suggests individual variability in source

35 reliability priors. The fact that we elicited prior estimates of different source type's reliability

36 before presenting the scenarios, and still find differences in the reliability estimates between the

37 control and retraction conditions, also demonstrates that, overall, participants are not solely

38 remaining consistent with their prior estimates of reliability. Finally, we observed a classic CIE

39 whereby misinformation endorsement ratings showed that a retraction, whether from the same or

40 a different source, did not bring endorsement ratings back to the baseline level (as shown in the

41 control condition). Participants continued to rely on retracted misinformation. Misinformation

42 probes were more strongly endorsed when misinformation was presented and retracted than

43 when the scenario did not involve a retraction of misinformation. This result is consistent with

44 previous CIE studies that have included a "no misinformation" control condition also find

45 baseline levels are higher than zero (e.g. Gordon et al., 2017; Johnson \& Seifert, 1994; Rich \&

46 Zaragoza, 2016). 


\section{THE RATIONAL CONTINUED INFLUENCE OF MISINFORMATION}

At the aggregate level, both posterior estimates for the hypothesis and misinformation endorsement ratings showed retention of the misinformation despite being retracted. The

49 posterior estimates for the hypothesis, while lower than the control condition, still showed

50 substantial retention of belief in the retracted hypothesis. It is worth noting that the posterior

51 probability estimates used in the present study measure belief updating and are therefore

52 qualitatively different from the traditional continued influence measures which measure

53 comprehension. Our novel probability estimate measures are, arguably, a more sensitive measure

54 of the CIE than traditional measures, as they demonstrate the uncertainty that often follows a

55 correction of the misinformation. People might reduce their belief in misinformation after a

56 retraction but not completely rule out the possibility that the misinformation is still valid because

57 they do not believe the retraction (Guillory \& Geraci, 2010, 2013; O'Rear \& Radvansky, 2020).

The present study did not include a condition in which misinformation is presented but

59 never retracted, as is common in most CIE research. The control and retraction conditions

60 sufficiently demonstrated higher endorsement in the retraction condition than the control

61 condition. Including a 'no retraction' condition would make it possible to directly compare the

62 novel approach used in the present study with previous CIE research and presents an opportunity

63 for follow-up research. The findings here also involve scenarios, and retractions, that are shorter

64 and more straightforward than the ones people may encounter in everyday life. Replicating the

65 findings with richer, more causally complex scenarios is necessary to establish whether the

66 modelling process still predicts the CIE.

67 Taken together we show that participants should exhibit the CIE (according to fitted BN

68 models), maintaining belief in the retracted misinformation. We found this effect with standard

69 behavioural measures used in the CIE literature (Brydges et al., 2018; Gordon et al., 2017), and 


\section{THE RATIONAL CONTINUED INFLUENCE OF MISINFORMATION}

70 observed retention of the hypothesis with novel probability estimate $\mathrm{P}(\mathrm{H})$ measures. We also

71 found appropriate penalization in reliability estimates given a contradiction among first and

72 second reporters - something hitherto unnoticed in CIE studies but predicted by our formalism.

73 An individual-level analysis of the data revealed that although many participants endorsed the

74 necessary assumptions for a rational CIE, very few of these participants went on to provide

75 posterior probability estimates in agreement with their model predictions. Furthermore, there was

76 only anecdotal evidence that participants whose BN predicted CIE also went on to endorse

77 misinformation. To put our findings in context with previous explanatory theories

78 (Lewandowsky et al., 2012), which tacitly assume that CIE is an error, we provide a process-

79 oriented theory that can give a rational (and testable) framework for CIE. We do not argue that

80 the sole explanation for the CIE relates to the inferences made about the reliability of sources

81 providing contradictory of information; instead, we argue that source reliability plays a crucial

82 role in the inferences that people generate after a correction to misinformation, and that there is a

83 richer context to consider when contemplating the CIE. We illustrate the (rationality-reversing)

84 impact of one such reasonable context expansion, but this is not to outright refute previous

85 descriptive theories per se.

86 To conclude, we provide a formal account of CIE using a BN framework and show that

87 CIE is in some circumstances, rational. This approach captures the qualitative inferences

88 participants make about the reliability of sources providing contradictory information and

89 suggests that perceived reliability moderates the degree to which people are willing to integrate

90 contradictory reports. The models described here are normative in the sense that they provide an

91 argument for why CIE can be the product of a rational process. We do not make the argument 


\section{THE RATIONAL CONTINUED INFLUENCE OF MISINFORMATION}

92 that the models describe participant reasoning itself. Finally, this research demonstrates that it is

93 possible to model CIE using a BN framework.

94 


\section{THE RATIONAL CONTINUED INFLUENCE OF MISINFORMATION}

\section{References}

AgenaRisk. In. (2019). Agena Ltd. http://www.agenarisk.com/

Bovens, L., \& Hartmann, S. (2003). Bayesian epistemology. Oxford University Press on Demand.

Brydges, C. R., Gignac, G. E., \& Ecker, U. K. (2018). Working memory capacity, short-term memory capacity, and the continued influence effect: A latent-variable analysis.

Intelligence, 69, 117-122.

Chater, N., Oaksford, M., Hahn, U., \& Heit, E. (2010). Bayesian models of cognition. Wiley Interdisciplinary Reviews: Cognitive Science, 1(6), 811-823.

Connor Desai, S., \& Reimers, S. (2019). Comparing the use of open and closed questions for Web-based measures of the continued-influence effect. Behavior research methods, 51(3), 1426-1440.

Cook, J., \& Lewandowsky, S. (2016). Rational irrationality: Modeling climate change belief polarization using Bayesian networks. Topics in cognitive science, 8(1), 160-179.

Corner, A., Hahn, U., \& Oaksford, M. (2011). The psychological mechanism of the slippery slope argument. Journal of Memory and Language, 64(2), 133-152.

Ecker, U. K., Lewandowsky, S., \& Apai, J. (2011). Terrorists brought down the plane!-No, actually it was a technical fault: Processing corrections of emotive information. The Quarterly Journal of Experimental Psychology, 64(2), 283-310.

Ecker, U. K., Lewandowsky, S., Cheung, C. S., \& Maybery, M. T. (2015). He did it! She did it! No, she did not! Multiple causal explanations and the continued influence of misinformation. Journal of Memory and Language, 85, 101-115.

Ecker, U. K., Lewandowsky, S., Swire, B., \& Chang, D. (2011). Correcting false information in memory: Manipulating the strength of misinformation encoding and its retraction. Psychonomic Bulletin \& Review, 18(3), 570-578.

Ecker, U. K., Lewandowsky, S., \& Tang, D. T. (2010). Explicit warnings reduce but do not eliminate the continued influence of misinformation. Memory \& cognition, 38(8), 10871100 .

Field, A. (2013). Discovering statistics using IBM SPSS statistics. sage.

Gordon, A., Brooks, J. C., Quadflieg, S., Ecker, U. K., \& Lewandowsky, S. (2017). Exploring the neural substrates of misinformation processing. Neuropsychologia, 106, 216-224. 


\section{THE RATIONAL CONTINUED INFLUENCE OF MISINFORMATION}

Guillory, J. J., \& Geraci, L. (2010). The persistence of inferences in memory for younger and older adults: Remembering facts and believing inferences. Psychonomic Bulletin \& Review, 17(1), 73-81.

Guillory, J. J., \& Geraci, L. (2013). Correcting erroneous inferences in memory: The role of source credibility. Journal of applied research in memory and cognition, 2(4), 201-209.

Hahn, U., Harris, A. J., \& Corner, A. (2009). Argument content and argument source: An exploration. Informal Logic, 29(4), 337-367.

Hahn, U., Harris, A. J., \& Oaksford, M. (2013). Rational argument, rational inference. Argument \& Computation, 4(1), 21-35.

Hahn, U., \& Oaksford, M. (2006). A normative theory of argument strength. Informal Logic, 26(1), 1-24.

Hahn, U., \& Oaksford, M. (2007). The rationality of informal argumentation: a Bayesian approach to reasoning fallacies. Psychological review, 114(3), 704.

Hahn, U., Oaksford, M., \& Bayindir, H. (2005). How convinced should we be by negative evidence. Proceedings of the 27th Annual Conference of the Cognitive Science Society,

Hahn, U., Oaksford, M., \& Corner, A. (2005). Circular arguments, begging the question and the formalization of argument strength. Proceedings of AMKLC'05, International symposium on adaptive models of knowledge, language and cognition,

Hahn, U., Oaksford, M., \& Harris, A. J. (2013). Testimony and argument: A Bayesian perspective. In Bayesian Argumentation (pp. 15-38). Springer.

Harris, A. J., \& Hahn, U. (2009). Bayesian rationality in evaluating multiple testimonies: Incorporating the role of coherence. Journal of Experimental Psychology: Learning, Memory, and Cognition, 35(5), 1366.

Harris, A. J., Hahn, U., Madsen, J. K., \& Hsu, A. S. (2016). The appeal to expert opinion: Quantitative support for a Bayesian network approach. Cognitive science, 40(6), 14961533.

Harris, A. J., Hsu, A. S., \& Madsen, J. K. (2012). Because Hitler did it! Quantitative tests of Bayesian argumentation using ad hominem. Thinking \& Reasoning, 18(3), 311-343.

Hayes, B. K., Banner, S., Forrester, S., \& Navarro, D. J. (2019). Selective sampling and inductive inference: Drawing inferences based on observed and missing evidence. Cognitive psychology, 113, 101221.

Ithisuphalap, J., Rich, P. R., \& Zaragoza, M. S. (2020). Does evaluating belief prior to its retraction influence the efficacy of later corrections? Memory, 28(5), 617-631. 


\section{THE RATIONAL CONTINUED INFLUENCE OF MISINFORMATION}

91

Jern, A., Chang, K.-M. K., \& Kemp, C. (2014). Belief polarization is not always irrational. Psychological review, 121(2), 206.

Johnson, H. M., \& Seifert, C. M. (1994). Sources of the continued influence effect: When misinformation in memory affects later inferences. Journal of Experimental Psychology: Learning, Memory, and Cognition, 20(6), 1420.

Lagnado, D. A., Fenton, N., \& Neil, M. (2013). Legal idioms: a framework for evidential reasoning. Argument \& Computation, 4(1), 46-63.

Lewandowsky, S., Ecker, U. K., \& Cook, J. (2017). Beyond misinformation: Understanding and coping with the "post-truth" era. Journal of applied research in memory and cognition, 6(4), 353-369.

Lewandowsky, S., Ecker, U. K., Seifert, C. M., Schwarz, N., \& Cook, J. (2012). Misinformation and its correction: Continued influence and successful debiasing. Psychological science in the public interest, 13(3), 106-131.

Madsen, J. K. (2016). Trump supported it?! A Bayesian source credibility model applied to appeals to specific American presidential candidates' opinions. CogSci,

Madsen, J. K., Hahn, U., \& Pilditch, T. D. (2018). Partial source dependence and reliability revision: the impact of shared backgrounds. CogSci,

Madsen, J. K., Hahn, U., \& Pilditch, T. D. (2020). The impact of partial source dependence on belief and reliability revision. Journal of Experimental Psychology: Learning, Memory, and Cognition.

O'Rear, A. E., \& Radvansky, G. A. (2020, Jan). Failure to accept retractions: A contribution to the continued influence effect. Mem Cognit, 48(1), 127-144. https://doi.org/10.3758/s13421-019-00967-9

Oaksford, M., \& Chater, N. (2007). Bayesian rationality: The probabilistic approach to human reasoning. Oxford University Press.

Oaksford, M., \& Hahn, U. (2004). A Bayesian approach to the argument from ignorance. Canadian Journal of Experimental Psychology/Revue canadienne de psychologie expérimentale, 58(2), 75.

Oaksford, M., \& Hahn, U. (2012). Why are we convinced by the ad hominem argument? Source reliability or pragma-dialectictics. Bayesian Argumentation, 39-58.

Pearl, J. (1988). Probabilistic reasoning in intelligent systems : networks of plausible inference. Morgan Kaufmann Publishers. 


\section{THE RATIONAL CONTINUED INFLUENCE OF MISINFORMATION}

137 Pilditch, T. D., Hahn, U., Fenton, N., \& Lagnado, D. (2020). Dependencies in evidential reports:

138 The case for informational advantages. Cognition, 204, 104343.

Pilditch, T. D., Hahn, U., \& Lagnado, D. A. (2018). Integrating dependent evidence: naïve reasoning in the face of complexity. CogSci,

Rich, P. R., \& Zaragoza, M. S. (2016). The continued influence of implied and explicitly stated misinformation in news reports. Journal of Experimental Psychology: Learning, Memory, and Cognition, 42(1), 62.

Schum, D. A. (1994). The Evidential Foundations of Probabilistic Reasoning. Northwestern University Press.

Schum, D. A., \& Martin, A. W. (1982). Formal and empirical research on cascaded inference in jurisprudence. Law and Society Review, 105-151.

Wilkes, A., \& Leatherbarrow, M. (1988). Editing episodic memory following the identification of error. The Quarterly Journal of Experimental Psychology, 40(2), 361-387.

Wilkes, A., \& Reynolds, D. (1999). On certain limitations accompanying readers' interpretations of corrections in episodic text. The Quarterly Journal of Experimental Psychology Section A, 52(1), 165-183. 